\title{
Effect of Laser-arc Hybrid Welding Energy Parameters on Welding Stability
}

\author{
ZHANG Fulong ${ }^{1,2,3}$, ZHANG Hong ${ }^{1,2,3 a}$ and LIU Fengde ${ }^{1,2,3}$ \\ ${ }^{1}$ College of Mechanical and Electric Engineering, Changchun University of Science and Technology, Changchun, Jilin Province, China \\ ${ }^{2}$ Engineering Research Center of Laser Processing for Universities of Jilin Province, Changchun University of Science and Technology, \\ Changchun, Jilin Province, China \\ ${ }^{3}$ National Base of International Science and Technology Cooperation in Optics, Changchun University of Science and Technology, \\ Changchun, Jilin Province, China
}

\begin{abstract}
The high-speed camera was used to collect the droplet transfer pattern and arc pattern of the laser-arc hybrid welding process. Using the methods of image processing and mathematical statistics, the effects of different laser and arc power conditions on the welding stability were studied. The results show that the melting width depends on the welding current, the depth of penetration depends on the laser power, the droplet transition pattern, the actual filament spacing and the arc length determine the welding stability of the laser arc hybrid welding.
\end{abstract}

\section{Introduction}

Laser-arc hybrid welding is a new welding mode consisting of a laser heat source and an arc heat source. It combines the advantages of laser welding and arc welding to avoid the disadvantages of both. At present, the laser-melting inert gas welding/metal active gas welding (MIG/MAG) hybrid welding technology has been widely studied and applied. In recent years, researchers have studied the mechanism of laser and arc based on spectral analysis, high-speed imaging, and welding electrical signal acquisition techniques [1]. Liu Liming et al. [2] studied the arc shape, electron temperature, and electron density of the YAG laser-TIG hybrid welding process. It was found that the laser keyhole formed on the surface of the laser is the key to the strength of the laser and arc. Li Zhiyong et al. [3] studied the heat-source coupling mechanism of laserMAG hybrid welding. The results show that the plasma and ultraviolet in the center of the welding arc are significantly enhanced after the laser is combined with MAG. In the front of the laser-induced arc, the broadening of the radiation interval of the corresponding spectral bands of FeI and FeII provides an ionization channel for the arc stabilization, which improves the laser weld formation and arc stability. T. Sibillano et al. [4,5] studied the correlation between plasma electron temperature and penetration depth during continuous $\mathrm{CO} 2$ laser and pulsed Nd:YAG laser welding. It was found that the electron temperature decreased with increasing laser energy.

The literature [6-8] studied the droplet transition frequency of laser-MAG/MIG hybrid welding, and pointed out that the large number of metallic plasmas generated by the laser action on the thermal radiation of the droplets promoted the droplet transfer. The attractive force of the plasma and the recoil force of the metal vapor on the droplet prevent the transition of the droplet to the weld pool, and the combination of the two changes the transition pattern and the transition frequency of the droplet.

In this paper, a high-speed camera is used to synchronously acquire the arc shape and droplet transfer characteristics of laser-arc hybrid welding. The droplet transfer mode of laser-arc hybrid welding is analyzed. This article provides reference data and theory for the practical application of laser-arc hybrid welding.

\section{Experimental method}

\subsection{Materials}

The test material was a $145 \mathrm{~mm} \times 30 \mathrm{~mm} \times 8 \mathrm{~mm}$ highnitrogen austenitic stainless steel. The wire model was H09Cr21Ni9MnMo with a diameter of $1.2 \mathrm{~mm}$. The surface of the workpiece was cleaned with acetone before welding.

\subsection{Welding equipments and conditions}

The test uses a Nd:YAG solid-state laser model HL4006D and a full-function digital MAG/MIG arc welder model YD-350Ag2HGE. The laser power was $3.0 \mathrm{~kW}$ and the wavelength was $1.06 \mu \mathrm{m}$. The laser was focused by a $223 \mathrm{~mm}$ focusing mirror and the spot diameter was $0.45 \mathrm{~mm}$ and the defocusing amount was $2 \mathrm{~mm}$. In the flat plate welding mode, the front laser is behind the arc in the welding direction, and the angle between the MAG welding gun and the laser beam is $30^{\circ}$.

*Corresponding author: h_zhangcust@126.com 
The MAG torch gas is $10 \% \mathrm{CO}_{2}+90 \%$ Ar and the gas flow rate is 15L.min-1. The experiment used a highspeed camera model CMOS-CR5000 $\times 2$ to synchronously record the arc shape and droplet transfer characteristics at a frequency of $5000 \mathrm{fps}$. The test device is shown in Figure 1. Welding parameters are shown in Table 1.

Table 1. Experimental parameters

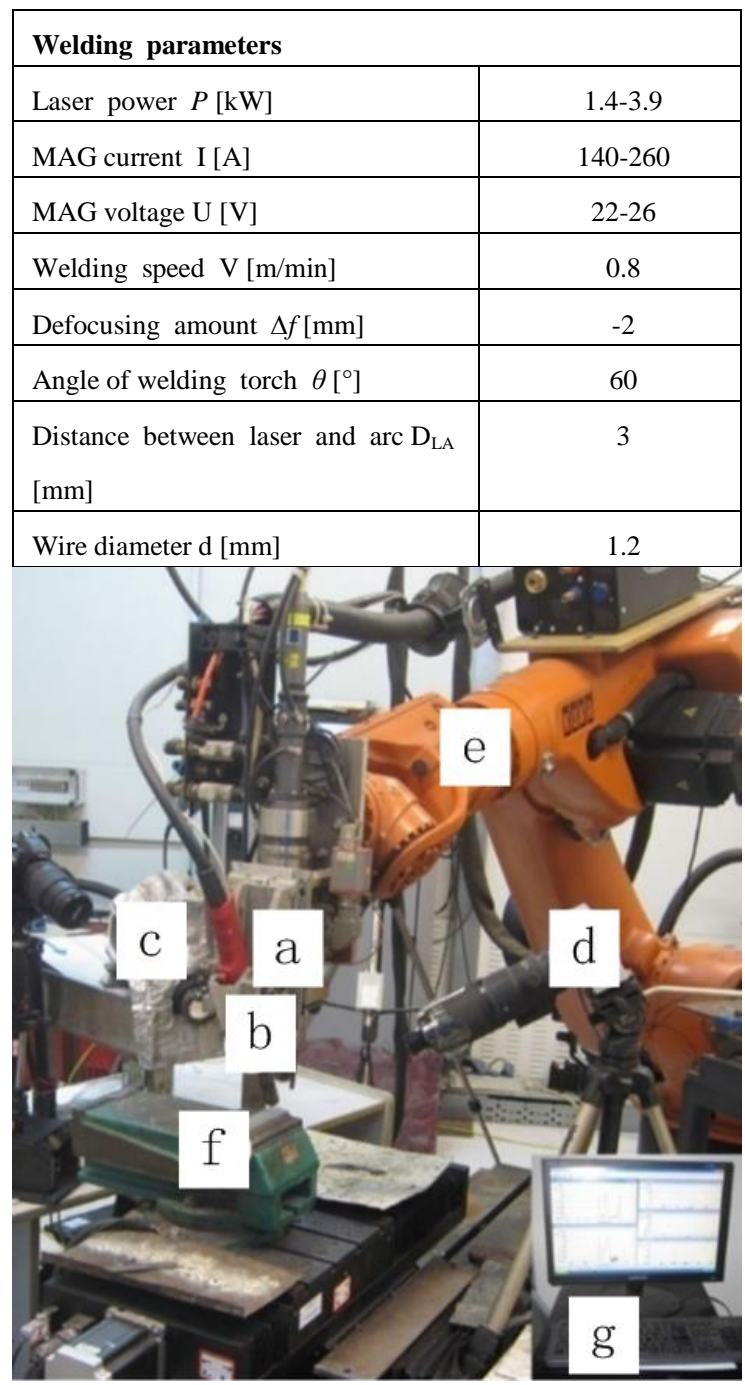

Figure 1. (a) Experimental setup: (a) laser beam; (b) MAG torch; (c) high speed camera-1; (d) high speed camera-2; (e) robot; (f)workpiece; (g) welding process analyzing and monitoring system.

\section{Results and discussion}

\subsection{Hybrid welding energy parameters on weld appearance}

Figure 2 shows the weld surface forming characteristics under the conditions of four different current parameters. It can be seen from Figure 2(a) that when the current is less than 170A, a large amount of splashing occurs on the weld surface, and the weld bead is not straight, due to the droplets. The transition is unstable, the weld height is inconsistent, the surface is rough, and there is a certain degree of undercut. When the current is in the range of 170A-190A, the weld surface splash gradually decreases. When the welding current is more than 190A, the weld seam is beautifully formed and the weld bead is even and straight. When the welding current exceeds $240 \mathrm{~A}$, the surface of the weld is heavily oxidized. As the current increases, the heat input increases, the area of the arc zone of the composite welding increases, and the section appearance of the weld transitions from a "nail" shape to an "umbrella" shape.

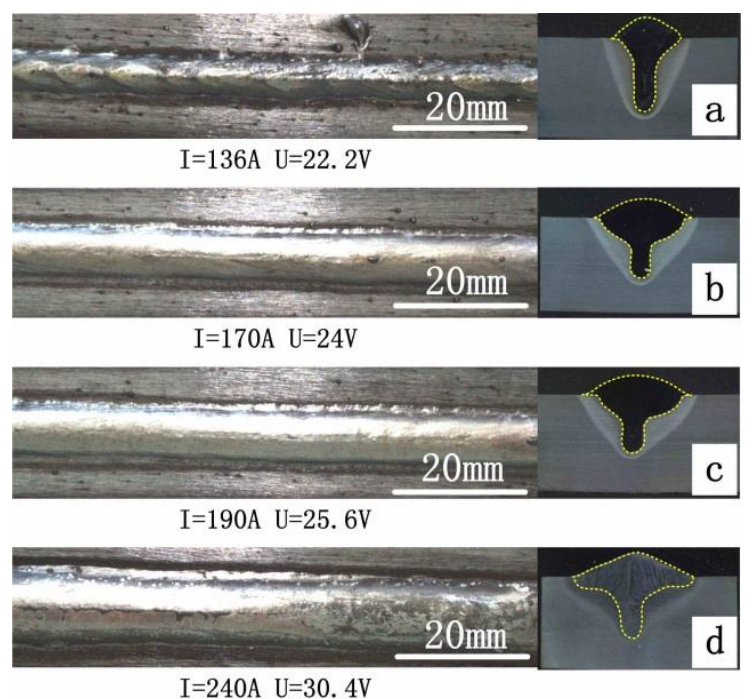

Figure 2 Different arc parameters on the surface and crosssection morphology of weld bead

Figure3, with gradually increasing welding current also increases bead width, weld width and the welding current linear fit. In laser-arc hybrid welding, the width of the weld is determined by the arc width of the arc. The keyhole generated by the laser on the surface of the workpiece draws and compresses the arc. As the laser energy increases,the keyhole is formed. The laser keyhole radiates energy outward to melt the keyhole. With increasing width, the laser power does not have a significant effect on the width of the weld as the welding current increases.

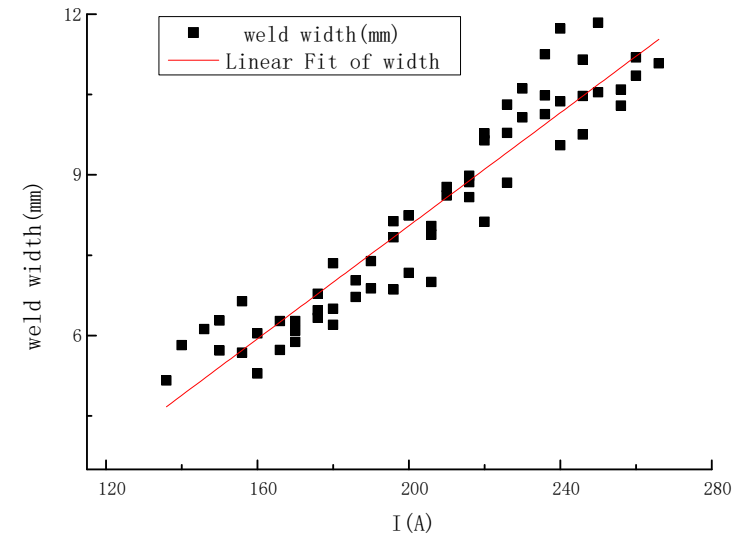

Figure 3 Relationships among current, Laser power and weld Width

Figure 4 shows the relationship between laser power, welding current and penetration depth. Polynomial fitting is used for the relationship between laser power and penetration depth. When the laser power is low,the 
welding current has a significant effect on the penetration depth. The welding current increases and the penetration depth increases. At this time, the laser energy enters the molten pool in the form of heat conduction, and is absorbed and reflected.As the laser power gradually increases, the arc plasma reversibly toughens to absorption, and a keyhole is formed on the surface of the workpiece to form a deep fusion welding mode. The penetration depth follows the laser light. Power increases significantly.

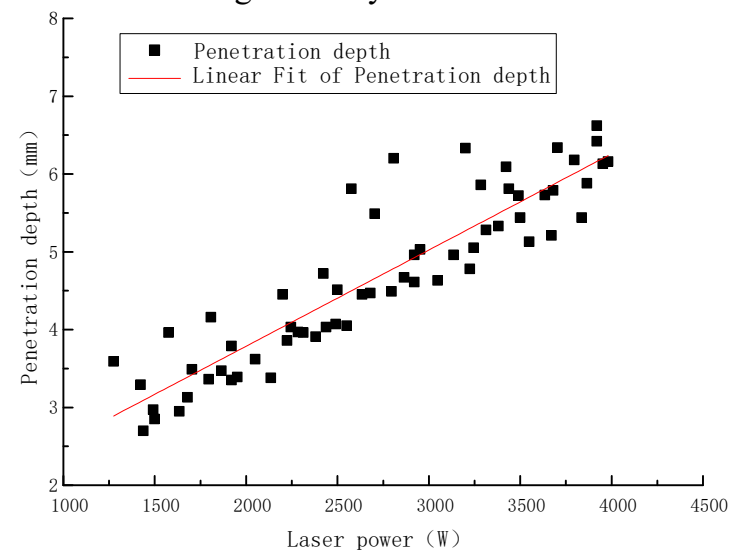

Figure 4. Relationships among Laser power, current and Weld width

Figure 5 shows the relationship between laser power and arc power and aspect ratio. When the power is relatively small, as shown in Figure 5(c), the composite effect of laser arc is not obvious. The cross-section of the weld is dominated by the shape of the individual arc welding, when the laser power When the power ratio is greater than 0.3 and the power ratio is greater than 0.3 , the characteristics of the hybrid welding gradually become obvious, as shown in Figure 5(b) as an 'umbrella' type. When the power ratio exceeds 1 , as shown in Figure (a), the characteristics of deep penetration welding are obvious.

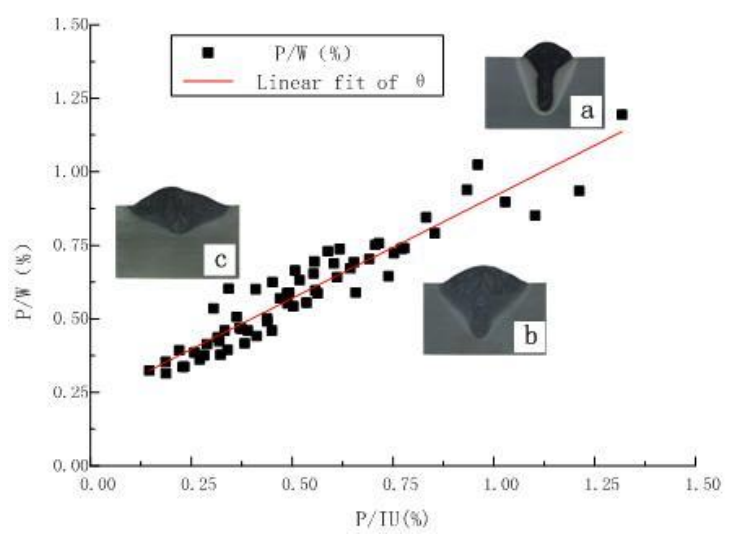

Figure 5. Relationship of laser power to arc power and depth to width

\subsection{Effect of Laser-arc welding energy parameter hybrid welding process stability}

According to the high-speed video capture of the image of the arc arc analysis, Figure 6 shows the relationship between voltage and arc length. The arc length is determined by the voltage. When the voltage is less than $24 \mathrm{~V}$ and the corresponding current value is less than 170A, as shown in Figure 7(a), the droplet transition mode is a short-circuit transition. The arc length changes greatly due to the instability of the short-circuit transition. When the voltage is low, as shown in Figure 7(b), the photo plasma increases the surface plasma concentration of the workpiece and reduces the arcing resistance. The arc forms a stable anode spot with the plasma generated by the laser keyhole, and the arc is compressed by the laser. The arc length is short; as the arc voltage increases, the arc length increases. As shown in Figure 7(c), the cross-sectional area of the arc cylinder increases. The arc absorbs more laser energy and causes the arc to expand. The stiffness of the arc increases to reduce the actual light. Wire spacing creates arc drift and affects the stability of the weld.

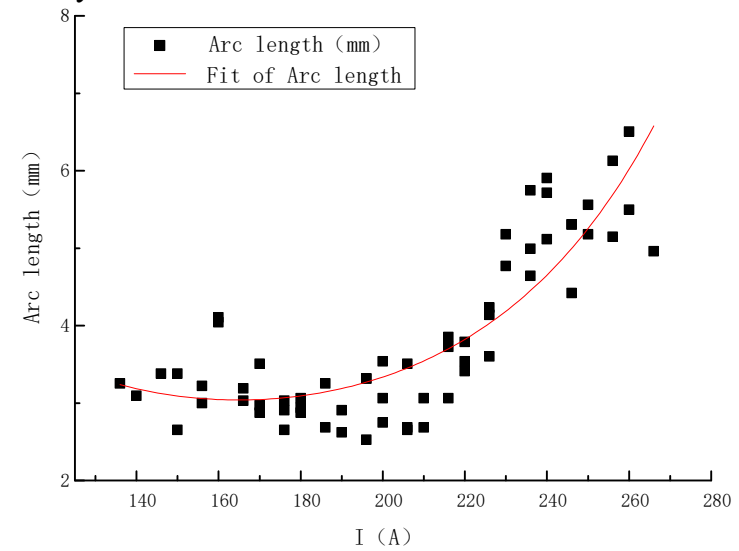

Figure 6. Relationship of welding voltage and Arc Length
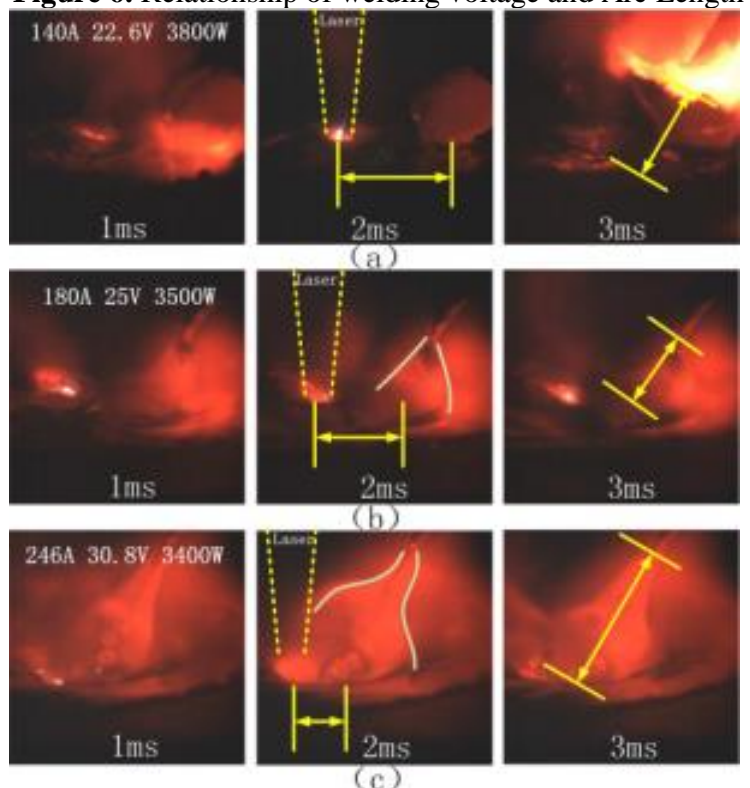

Figure 7. Different arc power parameters on the distance between laser and arc and arc length image

Figure 8 shows the relationship between the current and the actual filament pitch. From the figure, we can see that the actual filament pitch decreases with the increase of the welding current. When the current is small, as shown in Figure 7 (a), the actual light in the short-circuit state The wire pitch is the horizontal distance from the contact point of the droplet to the laser 
keyhole. At this time, the larger the droplet size, the actual light wire pitch is larger than the experimental preset value $(3 \mathrm{~mm})$, and the short-circuit transition causes a large fluctuation in the droplet size. The spacing is not stable. When the welding current is $180 \mathrm{~A}-240 \mathrm{~A}$, the actual optical fiber pitch fluctuates in a small range $(2.5 \mathrm{~mm}-3.5 \mathrm{~mm})$. At this time, the two heat sources have a significant composite effect, as shown in Figure 7(b). When the welding current exceeds 240A, as shown in Figure 7(c), the droplet transition mode is the transition of the droplet, the change of the force pattern of the droplet causes the change of the flying trajectory of the droplet, and the position of the droplet enters the molten pool and the position of the keyhole is compact, Some of the droplets interfered with the laser keyhole, hindering the role of laser energy in hybrid welding.

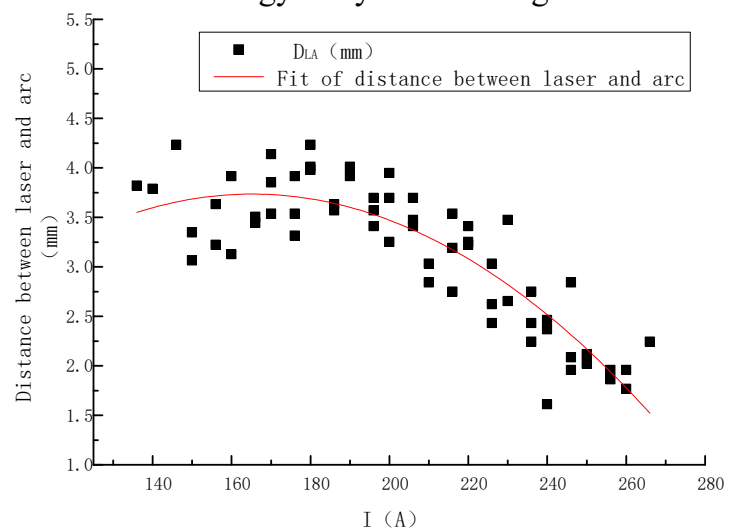

Figure 8. Relationship of current and distance between laser and arc

\section{Conclusion}

The melting width increases with the increase of the current, which is in line with the linear relationship. The laser power has little effect on the melting width; the penetration depth increases with the increase of the laser power and also meets the linear relationship, and the current does not have a great effect on the penetration depth. When the ratio of laser power to arc power is between 0.3 and 0.6 , the aspect ratio is in accordance with the characteristics of laser arc welding. When the arc length is about $3 \mathrm{~mm}$, the actual distance between laser and arc is $2.5 \mathrm{~mm}-3.5 \mathrm{~mm}$, laser arc welding is the best stability.

\section{References}

1. LIU S Y, CHEN S X, WANG Q H, et al. Analysis of plasma characteristics and conductive mechanism of laser assisted pulsed arc welding[J]. Optics and Lasers in Engineering, 2017, 92: 3947.

2. LIU L M, CHEN M H. Interactions between laser and arc plasma during laser-arc hybrid welding of magnesium alloy[J]. Optics and Lasers in Engineering, 2011, 49(9): 1224-1231.

3. ZHANG Wang, LI Fang, LI Min, et al. Study of the laser absorption characteristics of $\mathrm{CO}_{2}$ laser- pulsed GMAW hybrid welding based on spectral diagnosis technique[J]. Spectroscopy and Spectral Analysis, 2016, 36(4): 913-918.

4. LI Zhiyong, WANG Wei, WANG Xuyou, et al. Analysis of laser-MAG hybrid welding coupling mechanism based on spectrum[J]. Journal of Mechanical Engineering, 2010, 46(8): 62-67.

5. SIBILLANO T, ANCONA A, RIZZI D, et al. Study on the correlation between plasma electron temperature and penetration depth in laser welding processes[J]. Physics Procedia, 2010, 5(Part B): 429-436.

6. HU Lianhai, HUANG Jian, ZHUANG Kai, et al. Influence of distance between laser and MIG arc on drop transfer process of $\mathrm{CO} 2$ laser-MIG hybrid welding[J].Transactions of the china welding institution, 2010, 31(2): 49-53.

7. LIU S Y, LIU F D, ZHANG H, et al. Analysis of droplet transfer mode and forming process of weld bead in $\mathrm{CO} 2$ laser-MAG hybrid welding process[J]. Optics and Laser Technology, 2012, 44 (4) : 1019-1025.

8. [14] LIU S Y, LIU F D, XU C Y, et al. Experimental investigation on arc characteristic and droplet transfer in CO2 laser-metal arc gas (MAG) hybrid welding $[\mathrm{J}]$. International Journal of Heat and Mass Transfer, 2013, 62 (1): 604-611. 